\title{
The sustainable future of your dental practice
}

Manufacturers of dental supplies can make a huge difference when it comes to global warming by reducing greenhouse gases in their production. As a leading manufacturer of saliva ejectors, the Swedish company Orsing has launched a product line entirely manufactured from renewable resources.

Hygovac Bio is an aspirator tube made of nearly $100 \%$ bio-based polyethylene, a polyethylene entirely made from sugarcanes (Green PE). Unlike traditional polyethylene where fossil raw materials are used, sugarcanes are completely renewable. Hygovac Bio is available launched in two new lengths, $120 \mathrm{~mm}$

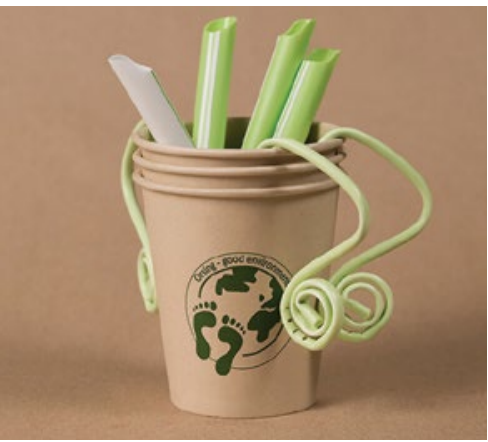

contact with the tongue and soft tissues and will not clog. Hygoformic Bio is seamlessly adjustable in shape and size to any patient's cheek and and $95 \mathrm{~mm}$, giving the user the opportunity to choose a length that suits the treatment. The aspirator tubes still have the same diameter and provide the same suction capacity as traditional Orsing's Hygovac products. The $95 \mathrm{~mm}$ version provides easier access for hygienists and dentists when working without assistance. Less material is required during manufacture.

The original mouldable saliva ejector with tongue holder used by dentists worldwide for more than 50 years is now also available in eco-friendly Green PE. Hygoformic Bio has a high suction capacity due to its five holes. The holes are placed inside the coil design to avoid of carbon dioxide per hectare per year, giving the plant a potentially crucial role in stabilising our planet's atmosphere.

Hygoformic Bio and Bio Adaptor are packed in Biodolomer, an innovative fully industrial compostable and biodegradable bioplastic certified according to EN13432 European standard. Biodolomer is made of calcium carbonate, sugar cane, rape seed and biodegradable PBS.

Orsing $\mathrm{AB}$ is a division of DirectaDentalGroup. Further information contact orsing@orsing.se or visitwww.orsing.se.

\section{Cutting-edge solutions for dental professionals}

There were plenty of exclusive offers and exciting product launches from W\&H at BDIA Dental Showcase 2019. The expert team demonstrated their latest and greatest solutions - from dental handpieces and surgical units, to new prophylaxis products and cutting-edge decontamination equipment.

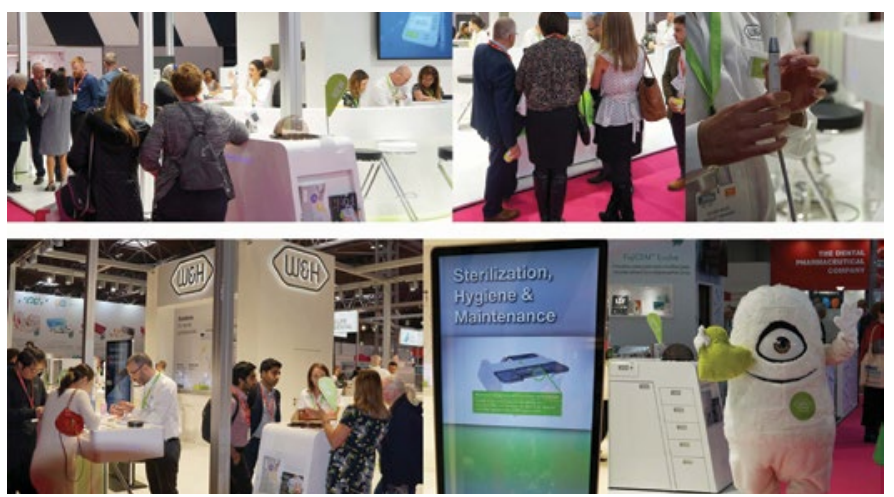

Delegates gained professional insight on best use of the Osstell Beacon, the Piezomed and the Implantmed with its innovative ISQ module. In addition, clinicians were able to get hands-on with the Proxeo Twist Cordless, the Proxeo Aura air polishing system, and Proxeo Ultra piezo scalers.

The new Lisa and Lara sterilisers also drew delegates to the stand with their intelligent, integrated features. Explore the full product range by contacting W\&H today.

To find out more visit www.wh.com/en_uk, call 01727874990 or email office.uk@wh.com.

\section{Attend a regional workshop and have your say}

The Faculty of General Dental Practice (FGDP[UK]) is urging dentists and dental care professionals throughout England to attend one of Health Education England's (HEE's) 'Advancing Dental Care' workshops.

Advancing Dental Care is HEE's review of dental education and training, which aims to improve NHS recruitment and retention, and address geographical and skills shortages. The aim of the events is to 'share the review's evidence and findings to date, to test thinking, and to develop and discuss options for flexible training for the dental workforce'.

There are 15 events taking place across the country between October 2019 and January 2020, each of which comes with certified CPD hours. The remaining sessions are:

- Tuesday 12 November 2019, 6.30-8.30pm, Cambridge

- Wednesday 13 November 2019, 6-8pm, Chester

- Thursday 14 November 2019, 5-7pm, London

- Tuesday 26 November 2019, 6-9pm, Slough

- Thursday 28 November 2019, 6-9pm, Eastleigh

- Wednesday 4 December 2019, 1-4pm, Leeds

- Tuesday 10 December 2019, 6-9.30pm, Bristol

- Wednesday 18 December 2019, 5.30-7.30pm, London

- Thursday 9 January 2020, 6-9.30pm, Plymouth.

Further information about the review is available at https:// www.hee.nhs.uk/our-work/advancing-dental-care/advancingdental-care-phase-ii, and further information on the workshops is available via the links above. 